\title{
Polylogarithmic Approximation for Euler Genus on Bounded Degree Graphs
}

\author{
Ken-ichi Kawarabayashi* \\ National Institute of Informatics \\ Tokyo, Japan \\ k_keniti@nii.ac.jp
}

\begin{abstract}
Computing the Euler genus of a graph is a fundamental problem in algorithmic graph theory. It has been shown to be NP-hard by [Thomassen '89, Thomassen '97], even for cubic graphs, and a lineartime fixed-parameter algorithm has been obtained by [Mohar '99]. Despite extensive study, the approximability of the Euler genus remains wide open. While the existence of an $O(1)$-approximation is not ruled out, the currently best-known upper bound is a $O\left(n^{1-\alpha}\right)$ approximation, for some universal constant $\alpha>0$ [Kawarabayashi and Sidiropoulos 2017]

We present an $O\left(\log ^{2.5} n\right)$-approximation polynomial time algorithm for this problem on graphs of bounded degree. Prior to our work, the best known result on graphs of bounded degree was a $n^{\Omega(1)}$-approximation [Chekuri and Sidiropoulos 2013].

As an immediate corollary, we also obtain improved approximation algorithms for the crossing number problem and for the minimum vertex planarization problem, on graphs of bounded degree. Specifically, we obtain a polynomial-time $O\left(\Delta^{2} \log ^{3.5} n\right)$ approximation algorithm for the minimum vertex planarization problem, on graphs of maximum degree $\Delta$. Moreover we obtain an algorithm which given a graph of crossing number $k$, computes a drawing with at most $k^{2} \log O(1) n$ crossings in polynomial time. This also implies a $n^{1 / 2} \log { }^{(1)} n$-approximation polynomial time algorithm. The previously best-known result is a polynomial time algorithm that computes a drawing with $k^{10} \log O(1)$ crossings, which implies a $n^{9 / 10} \log { }^{O(1)} n$-approximation algorithm [Chuzhoy 2011].
\end{abstract}

\section{CCS CONCEPTS}

- Theory of computation $\rightarrow$ Graph algorithms analysis; Approximation algorithms analysis.

*Supported by JST ERATO Kawarabayashi Large Graph Project JPMJER1201 and by JSPS Kakenhi JP18H05291.

${ }^{\dagger}$ Supported by NSF grants CCF- 1423230 and CCF-1815145, and by NSF CAREER award 1453472.

Permission to make digital or hard copies of all or part of this work for personal or classroom use is granted without fee provided that copies are not made or distributed for profit or commercial advantage and that copies bear this notice and the full citation on the first page. Copyrights for components of this work owned by others than ACM must be honored. Abstracting with credit is permitted. To copy otherwise, or republish, to post on servers or to redistribute to lists, requires prior specific permission and/or a fee. Request permissions from permissions@acm.org.

STOC '19, June 23-26, 2019, Phoenix, AZ, USA

(c) 2019 Association for Computing Machinery.

ACM ISBN 978-1-4503-6705-9/19/06 . \$15.00

https://doi.org/10.1145/3313276.3316409

\author{
Anastasios Sidiropoulos ${ }^{\dagger}$ \\ Dept. of Computer Science, University of Illinois at \\ Chicago \\ Chicago, USA \\ sidiropo@uic.edu
}

\section{KEYWORDS}

graph genus, approximation algorithms, crossing number

ACM Reference Format:

Ken-ichi Kawarabayashi and Anastasios Sidiropoulos. 2019. Polylogarithmic Approximation for Euler Genus on Bounded Degree Graphs. In Proceedings of the 51st Annual ACM SIGACT Symposium on the Theory of Computing (STOC '19), June 23-26, 2019, Phoenix, AZ, USA. ACM, New York, NY, USA, 12 pages. https://doi.org/10.1145/3313276.3316409

\section{INTRODUCTION}

In this paper we consider the following basic problem: Given a graph $G$, compute its Euler genus, denoted by $\operatorname{eg}(G)$. This is a fundamental problem in algorithmic graph theory, which was emphasized by Garey and Johnson [5], and first shown to be NP-hard by Thomassen $[29,30]$.

Part of the original motivation for the study of the genus of graphs goes back to the Heawood problem during the 19th century, which concerns the maximum chromatic number of graphs embeddable in a fixed surface. The solution of the Heawood problem turned out to be equivalent to determining the genus of complete graphs (cf. [22]).

Apart from bounds on the genus of specific families of graphs, there are no general results available. Nevertheless, many related problems have been studied extensively. For example, a seminal result of Hopcroft and Tarjan [7] asserts that there is a linear time algorithm for testing planarity of graphs, and for computing a planar embedding if one exists. This algorithm has received considerable amount of attention in both theory and practice. A natural generalization of the problem of computing a planar embedding is the problem of embedding a graph into a surface of fixed genus $g$. From the work of Robertson and Seymour [26], the family of graphs of genus at most $g$ is characterized as the class of graphs that excludes as a minor all graphs from a finite family. This implies that the problem is fixed-parameter tractable, parameterized by $g$. However, the family of excluded minors is not known explicitly even for small Euler genus $g$; it contains two graphs for $g=0$, which is Kuratowski's theorem [14,31], and 35 graphs for $g=1[1,6]$. Moreover, the number of excluded minors for Euler genus $g$ is exponential in terms of $g$. Linear time algorithms have been devised for embedding graphs into the projective plane [16] and the torus [8]. Mohar $[17,18]$ finally gave a linear time algorithm for embedding a graph into an arbitrary fixed surface. This is one of the deepest results in this area. A relatively simple linear-time algorithm was given by Kawarabayashi, Mohar, and Reed [10]. This result has recently been further generalized by Kociumaka and Pilipczuk [13] to obtain 
a linear-time algorithm for deleting a fixed-size set of vertices to obtain a graph of fixed genus. We remark that the dependence of the running time of all of the above mentioned exact algorithms is at least exponential in $g$.

Approximating genus and our result. We consider the problem of approximating $\operatorname{eg}(G)$, for some given graph $G$, when $\operatorname{eg}(G)$ is not fixed. Perhaps surprisingly, despite its central importance, very little was known for this problem on general graphs until quite recently. Let us first briefly describe what is currently known. The bound on Euler's characteristic implies that any $n$-vertex graph of Euler genus $g$ has at most $O(n+g)$ edges. Since any graph can be drawn in a surface that has one handle for every edge, this immediately implies a $\mathrm{O}(n / g)$-approximation, which is a $\Theta(n)$-approximation in the worst case. Mohar has obtained a $O(1)$-approximation for graphs $G$ that contain a vertex $a$ such that $G-a$ is planar and 3-connected (note that this is a special class of 1-apex graphs). Makarychev, Nayyeri, and Sidiropoulos [15] obtained an algorithm that given a Hamiltonian graph $G$ along with a Hamiltonian path $P$, computes an embedding of $G$ in a surface of Euler genus $g^{O(1)} \log O(1) n$ where $g$ is the orientable genus of $G$. We remark that the Hamiltonicity assumption is a major restriction of this algorithm.

On the lower-bound side, Mohar [19] showed that computing $\operatorname{eg}(G)$ remains NP-hard even when the input is a 1-apex graph. We emphasize that essentially no inapproximability result is known for $\operatorname{eg}(G)$, even on graphs of bounded degree. In other words, we currently cannot exclude the existence of an $O(1)$-approximation.

Recently, some nontrivial approximation results appeared. Chekuri and Sidiropoulos [2] have obtained a polynomial-time algorithm which given a graph $G$ of maximum degree $\Delta$ computes an embedding of $G$ in a surface of Euler genus at most $(\Delta \cdot \operatorname{eg}(G))^{O(1)} \log { }^{O(1)} n$. This result implies a polynomial-time $O\left(n^{1-\alpha}\right)$-approximation algorithm for Euler genus, for some universal constant $\alpha>0$. Both of these results have subsequently been extended to arbitrary graphs (i.e. without the bounded degree condition) by Kawarabayashi and Sidiropoulos [11].

In this paper, we significantly improve the result of Chekuri and Sidiropoulos [2], by showing the following.

Theorem 1.1 (PolylogarithmiC APPROXIMATION ALgORITHM FOR EULER GENUS ON BOUNDED DEGREE GRAPHS). There exists a $O\left(\log ^{2.5} n\right)$-approximation algorithm for the genus problem on graphs of bounded degree. More precisely, given a graph $G$ of Euler genus $g$ and of maximum degree $\Delta$, we can compute, in polynomial time, an embedding of $G$ into a surface of Euler genus $O\left(\Delta^{2} \cdot g \cdot \log ^{2.5} n\right)$.

It would be of great interest to remove the degree condition in Theorem 1.1, but our proof does use this condition extensively. So it seems that we need some further new ideas. One serious obstacle is that in our proof, we have to embed a $k$-apex graph in a surface, where $k=O\left(\operatorname{eg}(G) \cdot \log ^{c} n\right)$ for some absolute constant $c$. For bounded-degree graphs, this can be done trivially (see below), but without the degree condition, this seems challenging.

The above result has immediate applications for the minimum planarization and crossing number problems on graphs of bounded degree. For some graph $G$, let $\operatorname{mvp}(G)$ denote the minimum cardinality of some $X \subset V(G)$, such that $G \backslash X$ is planar. Clearly, for graphs of maximum degree $\Delta$, we have $\operatorname{eg}(G) \leq \Delta \cdot \operatorname{mvp}(G)$. Thus
Theorem 1.1 can be used to compute an embedding into a surface of Euler genus at most $O\left(\Delta^{3} \operatorname{mvp}(G) \log ^{2.5} n\right)$. It is shown in [12] that given an embedding of $G$ into some surface of genus $\gamma$, one can compute, in polynomial time, a set $X \subset V(G)$, such that $G \backslash X$ is planar, with $|X|=O\left(\gamma \log n+\operatorname{mvp}(G) \log ^{2} n\right)$. We thus immediately obtain the following:

Corollary 1.2. There exists a polynomial-time $O\left(\Delta^{3} \log ^{3.5} n\right)$-approximation algorithm for the minimum vertex planarization problem, on graphs of maximum degree $\Delta$.

Combining the above result with the techniques from [4] we obtain the following algorithm for the crossing number problem (see [12] for details).

COROLlary 1.3. There exists a polynomial-time $\Delta^{O(1)} n^{1 / 2} \log { }^{O(1)} n$-approximation algorithm for the crossing number problem, on graphs of maximum degree $\Delta$.

Prior to our work, the best-known result was a $n^{1 / 2+\varepsilon}$-approximation in time $\Delta^{O(1)} n^{O(1 / \varepsilon)}$ and a $\Delta^{O(1)} n^{1 / 2} \log { }^{O(1)}$-approximation in time $n^{O(\log n / \log \log n)}[12]$.

\subsection{High Level Description of the Algorithm}

We now give a high-level description of our approach. Our algorithm is inspired by and builds upon the approximation algorithm due to Chekuri and Sidiropoulos [2], and the recent approximation algorithm for the minimum planarization problem due to Kawarabayashi and Sidiropoulos [12].

Ideas from prior work. The approach from [2] is based on ideas from the fixed-parameter case and the theory of graph minors $[10,24,28]$. However, the implementation of certain steps from the exact case is quite challenging due to the fact that the parameters are not fixed in the approximate setting.

The algorithm of [2] proceeds as follows. First, while the input graph has sufficiently large treewidth, it finds a subgraph that can be removed without significantly affecting the solution. This is done by computing a flat grid minor. A subgraph $H \subset G$ is flat if it is planar and there exists a planar drawing of $H$ where all vertices in $H$ that are incident to $G \backslash H$ lie in the outer face. Eventually, it arrives at a graph of small treewidth. For such a graph they can compute a small set of vertices $X$ whose removal leaves a planar graph. Since the degree is bounded, they can add $X$ back to the planar graph by introducing at most a constant number of handles for every vertex in $X$.

This approach does not yield a polylogarithmic approximation, because in order to compute a sufficiently large flat grid minor, the treewidth has to be at least $\Omega\left(g^{c}\right)$ for some $c>1$. This implies that the set $X$ of deleted vertices has size $\Omega\left(g^{c}\right)$, and thus the total number of handles needed to extend the embedding to $X$ is $\Omega\left(g^{c}\right)$. In order to obtain a polylogarithmic approximation, we need to be able to handle graphs of treewidth $g \log ^{O(1)} n$, which is the main challenge, as we shall see below.

Overview of our algorithm. We now present an overview of our algorithm. Given a graph $G$ of maximum degree $\Delta=O(1)$ with $\operatorname{eg}(G)=g$, the algorithm proceeds as follows.

1. The small treewidth case. If the treewidth is $O(g \log n)$, then we can find some separator $X$ of size $O\left(g \log ^{1.5} n\right)$, and 
we recurse on all non-planar connected components of $G \backslash X$. It can be shown that $|X|$ can be charged to the Euler genus $g$, so that the total number of vertices in all the balanced separators removed throughout the recursion is at most $O\left(g \log ^{2.5} n\right)$

It thus remains to consider the case when the treewidth of $G$ is large. We now give a brief overview of our approach and highlight some of the main challenges.

2. The large treewidth case: Existence of $\Omega(\log n)$ nested cycles. If the treewidth of $G$ is at least $\Omega(g \log n)$, then we show that in the optimal embedding of $G$ in this surface, there are $\Omega(\log n)$ "nested" cycles. We remark that we only need this existential result, and we do not compute such nested cycles. The proof of this fact is based on tools and ideas due to Reed [21] that were used to find an irrelevant vertex for the disjoint paths problem on planar graphs (see also [25, 27]).

3. Computing $\Omega(\log n)$ nested cycles and irrelevant vertices. From Step 2 we are guaranteed that if the treewidth is $\Omega(g \log n)$, then there exist $\Omega(\log n)$ nested cycles. We next show the following: any vertex $v$ which is surrounded by these nested cycles is irrelevant in the sense that the Euler genus of $G-v$ is the same as that of $G$, and moreover, if we have an embedding of $G-v$ in a surface of Euler genus $g$, then we can extend it to $G$ in the same surface. Moreover, we give a polynomial time algorithm to find such a vertex $v$ if one exists.

4. Embedding into a higher genus surface. Given the above procedure for finding an irrelevant vertex, the rest of our algorithm proceeds as follows. We iteratively compute one of the following (1) a small balanced separator (from Step 1), or (2) a set of irrelevant vertices (from Step 3). The graph obtained at the end of this sequence is planar. Let $X^{\prime}$ be the set of vertices removed in case (1), throughout the execution. From Step 1, we have $\left|X^{\prime}\right|=O\left(g \log ^{2.5} n\right)$. We say that the cost of the sequence is $\left|X^{\prime}\right|$.

However, the number of irrelevant vertices removed in (2) can be as large as $\Omega(n)$. We need to add these vertices to the planar drawing of the resulting graph. It turns out that this is not always possible. The reason is that the irrelevant vertices removed are only guaranteed to be irrelevant w.r.t. any optimal embedding in a surface of Euler genus eg $(G)$.

We overcome this obstacle using a technique that was first introduced in [3]: When deleting a set of irrelevant vertices, we add a grid of width 3, referred to as a frame, around the "hole" that is created (see Figure 1). The point of adding this grid is that we can inductively add the irrelevant vertices back to the graph as follows: If $X^{\prime}$ does not intersect the frame, then we can simply add the irrelevant vertices back to the graph without violating planarity. Otherwise, if the frame intersects $m$ vertices from $X^{\prime}$, then we can extend the current drawing to the irrelevant vertices corresponding to that frame by adding at most $m$ handles or antihandles. This leads to an embedding into a new non-planar surface. Repeating this process over all frames, we obtain an embedding of $G \backslash X^{\prime}$ into some surface of Euler genus $O\left(g \log ^{2.5} n\right)$.
5. The final algorithm. The last remaining step is to extend the embedding to all the vertices in $X^{\prime}$. Note that at this point, the graph $G \backslash X^{\prime}$ is embedded into some surface of Euler genus $O\left(g \log ^{2.5} n\right)$. Since the maximum degree is $\Delta$, for each vertex $v \in X^{\prime}$, we can trivially extend the embedding to $v$ by adding at most $\Delta=O(1)$ handles to the surface.

As we see here, Steps 1 and 5 are trivial. Step 4 is very similar to that given in [3]. Step 3 is an algorithmic application of Step 2. The main technical challenge in the above approach is Step 2 and its algorithmic implementation in Step 3. These steps are presented in Sections 4 and 5, respectively.

\subsection{Organization}

The rest of the paper is organized as follows. Section 2 introduces some notation and preliminary results used throughout the paper. Section 3 presents the main algorithm. Section 4 gives the proof that any graph of sufficiently large treewidth contains an isolated vertex. Section 5 shows that isolated vertices are irrelevant. Section 6 gives a polynomial-time algorithm for computing an isolated vertex.

\section{DEFINITIONS AND PRELIMINARIES}

In this section we introduce some notation used throughout the paper.

Graph notation. Let $G$ be a graph and $X \subseteq V(G)$. We use $d_{G}$ to denote the shortest path metric on $G$. For any $v \in V(G)$ we write $d_{G}(X, v)=d_{G}(v, X)=\min _{u \in X} d_{G}(v, u)$. For any $r \geq 0$ we define $N_{G}(X, r)=\left\{v \in V(G): d_{G}(v, X) \leq r\right\}$. For a simple path $P$ and $u, v \in V(P)$ we denote by $P[u, v]$ the subpath of $P$ between $u$ and $v$. We define the $(r \times \ell)$ grid to be the Cartesian product $P_{r} \times P_{\ell}$, where $P_{i}$ denote the path with $i$ vertices.

Let $K$ be a subgraph of $G$. A $K$-bridge $B$ in $G$ (or a bridge $B$ of $K$ in $G)$ is a subgraph of $G$ which is either an edge $e \in E(G) \backslash E(K)$ with both endpoints in $K$, or it is a connected component of $G \backslash K$ together with all edges (and their endpoints) between the component and $K$. The vertices of $B \cap K$ are the attachments of $B$.

Graph on surfaces. A drawing of a graph $G$ into a surface $\mathcal{S}$ is a mapping $\phi$ that sends every vertex $v \in V(G)$ into a point $\phi(v) \in \mathcal{S}$ and every edge into a simple curve connecting its endpoints, so that the images of different edges are allowed to intersect only at their endpoints. The Euler genus of a surface $\mathcal{S}$, denoted by eg $(\mathcal{S})$, is defined to be $2-\chi(\mathcal{S})$, where $\chi(\mathcal{S})$ is the Euler characteristic of $\mathcal{S}$. This parameter coincides with the usual notion of genus, except that it is twice as large if the surface is orientable. For a graph $G$, the Euler genus of $G$, denoted by $\operatorname{eg}(G)$, is defined to be the minimum Euler genus of a surface $\mathcal{S}$, such that $G$ can be embedded into $\mathcal{S}$.

Let $G$ be a graph and let $\phi$ be an embedding of $G$ into some surface $\mathcal{S}$. A simple non-contractible loop $\gamma$ in $\mathcal{S}$ is called a $\phi$-noose if it intersects the image of $G$ only on vertices. We define the length of $\gamma$ to be len $(\gamma)=|\{v \in V(G): \phi(v) \in \gamma\}|$.

Let $G$ be a graph, $v \in V(G)$, and let $C$ be the set of connected components of $G \backslash v$. We say that the graph $G^{\prime}=\bigsqcup_{C \in C} G[C \cup v]$ is obtained by cutting $G$ along $v$, where $\sqcup$ denotes disjoint union. For a set $X=\left\{v_{1}, \ldots, v_{k}\right\} \subseteq V(G)$ we say that a graph $G^{\prime}$ is obtained by cutting $G$ along $X$ if there exists a sequence of graph $G_{0}, \ldots, G_{k}$ 
with $G_{0}=G, G_{k}=G^{\prime}$, and such that for each $i \in\{1, \ldots, k\}$ the graph $G_{i}$ is obtained by cutting $G_{i-1}$ along $v_{i}$.

A graph $G$ embedded in a surface $S$ has representativity at least $k$, if every non-contractible closed curve in the surface intersects the graph in at least $k$ points. This notion turns out to be of great importance in topological graph theory, cf. [20].

Whitney proved that any embedding of a graph in the sphere can be obtained from any other embedding of this graph into the sphere by performing a sequence of simple local re-embeddings, called Whitney flips. See, e.g [20] for more details. These changes are non-trivial only when the graph is not 3-connected. Whitney changes are defined for embeddings in arbitrary surfaces. We say that two embeddings of the same graph $G$ are Whitney equivalent if one embedding can be obtained from the other by a sequence of Whitney flips. Here, this flip only happens in a planar way, i.e., Whitney flips do not happen if there is a non-contractible curve of order at most two.

Treewidth. We use $\operatorname{tw}(G)$ to denote the treewidth of $G$. We need the following result for planar graphs.

Lemma 2.1 (Robertson et AL. [23]). Any planar graph $G$ contains some $(r \times r)$-grid minor for some $r=\Omega(\operatorname{tw}(G))$.

\section{THE MAIN ALGORITHM}

In this Section we present the main algorithm for approximating genus. We first introduce some definitions. Intuitively, a patch is a small irrelevant subgraph, that is contained inside some disk in any optimal solution. The framing of a patch is a new graph, that does not contain the interior of the patch, and instead contains a grid of constant width attached to the boundary of the patch. Computing a framing in this manner will allow us to extend an approximate solution to the whole graph by embedding into a surface of slightly higher genus.

Definition 3.1 (РАтсH). Let $G$ be a graph, let $\Gamma \subseteq G$, and let $C \subseteq \Gamma$ be a cycle. Suppose that there exists a planar drawing of $\Gamma$ having $C$ as the outer face. Then we say that $(\Gamma, C)$ is a patch (of $G$ ).

We say that two patches $(\Gamma, C),\left(\Gamma^{\prime}, C^{\prime}\right)$ of $G$ are interior-disjoint if $V(\Gamma) \cap V\left(\Gamma^{\prime}\right)=V(C) \cap V\left(C^{\prime}\right)$.

Definition 3.2 (Framing). Let $G$ be a graph and let $(\Gamma, C)$ be a patch of $G$. Suppose that $V(C)=\left\{v_{0}^{0}, \ldots, v_{t-1}^{0}\right\}$, and $E(C)=$ $\left\{\left\{v_{0}^{0}, v_{1}^{0}\right\}, \ldots,\left\{v_{t-2}^{0}, v_{t-1}^{0}\right\},\left\{v_{t-1}^{0}, v_{0}^{0}\right\}\right\}$. Let $G^{\text {framed }}$ be the graph with $V\left(G^{\text {framed }}\right)=(V(G) \backslash(V(\Gamma) \backslash V(C))) \cup \bigcup_{i=1}^{t-1} \cup_{j=1}^{3}\left\{v_{i}^{j}\right\}$, where $v_{0}^{j}, \ldots, v_{t-1}^{j}$ are new vertices, and

$$
\begin{aligned}
E\left(G^{\text {framed }}\right)= & (E(G) \backslash(E(\Gamma) \backslash E(C))) \\
& \cup\left(\bigcup_{i=0}^{t-1} \bigcup_{j=0}^{2}\left\{\left\{v_{i}^{j}, v_{i}^{j+1}\right\},\left\{v_{i}^{j}, v_{i+1 \bmod t}^{j}\right\}\right\}\right) .
\end{aligned}
$$

We refer to the graph $G^{\text {framed }}$ as the $(\Gamma, C)$-framing of $G$ (see Figure 1 for an example).

Let $\mathcal{P}=\left(\Gamma_{1}, C_{1}\right), \ldots,\left(\Gamma_{k}, C_{k}\right)$ be a sequence of pairwise interiordisjoint patches of $G$. Let $G_{0}=G$, and for all $i \in\{1, \ldots, k\}$, let $G_{i}$ be the $\left(\Gamma_{i}, C_{i}\right)$-framing of $G_{i-1}$. Then we say that $G_{k}$ is the $\mathcal{P}$ framing of $G$. We remark that the $\mathcal{P}$-framing of $G$ is invariant under permutations of $\mathcal{P}$.
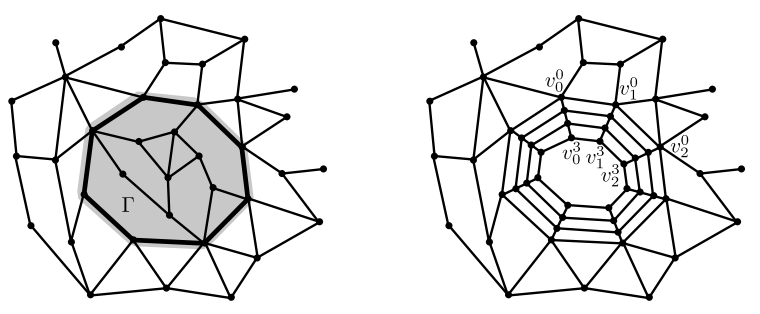

Figure 1: A patch $(\Gamma, C)$ in a graph $G$, where the shaded area contains the graph $\Gamma$, and the cycle $C$ is drawn in bold (left), and the $(\Gamma, C)$-framing of $G$ (right).

Using the above notions, we can now define the concept of a trimming sequence. Intuitively, this consists of a sequence of operations that inductively simplify the graph until it becomes planar.

Definition 3.3 (Trimming Sequence). Let $G$ be a graph. Let $\mathcal{G}=\left(G_{0}, A_{0}\right), \ldots,\left(G_{\ell}, A_{\ell}\right)$ be a sequence satisfying the following properties:

(1) For all $i \in\{0, \ldots, \ell\}, G_{i}$ is a graph. Moreover $G_{0}=G$ and $G_{\ell}$ is planar.

(2) For all $i \in\{1, \ldots, \ell\}$, exactly one of the following holds: (2.1) $A_{i-1} \subset V\left(G_{i-1}\right)$ and $G_{i}=G_{i-1} \backslash A_{i-1}$. We say that $i-1$ is a deletion step (of $\mathcal{G}$ ).

(2.2) $A_{i-1}$ is a sequence of pairwise interior-disjoint patches of $G_{i-1}$, and $G_{i}$ is the $A_{i-1}$-framing of $G_{i-1}$. We say that $i-1$ is a framing step (of $\mathcal{G}$ ).

We also let $A_{\ell}=\emptyset$.

We say that $\mathcal{G}$ is a trimming sequence (for $G$ ). We also define the cost of $\mathcal{G}$ to be $\operatorname{cost}(\mathcal{G})=\sum_{i \in\{0, \ldots, \ell-1\}: i \text { is a deletion step }}\left|A_{i}\right|$.

The next Lemma shows how to compute a trimming sequence of low cost. Due to lack of space, its proof is appears in the full version of this paper.

LEMma 3.4 (COMPUTING A TRIMMing SEQUence). Let $G$ be an $n$-vertex graph of maximum degree $\Delta$ and with $\operatorname{eg}(G)=g$. Then, in polynomial time, we can compute a trimming sequence $\mathcal{G}$ with $\operatorname{cost}(\mathcal{G})=O\left(\Delta \cdot g \cdot \log ^{2.5} n\right)$.

The next Lemma shows that given a trimming sequence of low cost, we can efficiently compute an embedding into a surface of low Euler genus, after deleting a small number of vertices. The proof of this result was given in [12], where it was used in the minimum planarization problem. We provide a proof in the full vesion of this paper.

LEMMA 3.5 (EMBEDdING INTO A HIGHER GENUS SURFACE). Let $G$ be a graph and let $\mathcal{G}$ be a trimming sequence for $G$. Then there exists a polynomial time algorithm which given $G$ and $G$ outputs some $X \subseteq V(G)$, with $|X| \leq \operatorname{cost}(G)$, and an embedding of $G \backslash X$ in some surface of Euler genus $\operatorname{eg}(G \backslash X)=O(\operatorname{cost}(\mathcal{G}))$.

We are now ready to present our main results in this paper, which combines Lemmas 3.4 and 3.5. 
Proof of Theorem 1.1. By Lemma 3.4 we can compute a trimming sequence. By Lemma 3.5 there exists a polynomial time algorithm which outputs some $X \subseteq V(G)$, with $|X| \leq \operatorname{cost}(\mathcal{G})=$ $O\left(\Delta \cdot g \cdot \log ^{2.5} n\right)$, and an embedding of $G \backslash X$ in some surface of $\operatorname{eg}(G \backslash X)=O\left(\Delta \cdot g \cdot \log ^{2.5} n\right)$. For each vertex of $X$, we can add at most $\Delta$ handles to embed it to the surface. Thus we obtain an embedding of $G$ in a surface of Euler genus $O\left(\Delta^{2} \cdot g \cdot \log ^{2.5} n\right)$.

\section{EXISTENCE OF AN ISOLATED VERTEX}

In this section we show that if the treewidth is sufficiently large, then there exists a large sequence of "nested" cycles. Let $G$ be a graph and let $v \in V(G)$. We say that $v$ is $\ell$-isolated if there are vertex disjoint cycles $C_{1}, \ldots, C_{\ell}$ of $G$ such that each $C_{i}$ separates $G$ with all $C_{j}$ in one component of $G \backslash C_{i}$ (for $j<i$ ) and all other cycles in the other component of $G \backslash C_{i}$, and moreover, if we let $G^{\prime}$ be the connected component of $G \backslash C_{\ell}$ that contains $v$, then there exists a planar embedding of $G\left[V\left(C_{\ell}\right) \cup V\left(G^{\prime}\right)\right]$, such that $C_{1}, \ldots, C_{\ell}$ bound disks $D_{1}, \ldots, D_{\ell}$, with $v \in D_{1} \subset D_{2} \subset \ldots, \subset D_{\ell}$. We say that the cycles $C_{1}, \ldots, C_{\ell}$ are $\ell$-nested. When the sequence $C_{1}, \ldots, C_{\ell}$ is clear from the context, we shall refer to $C_{1}$ and $C_{\ell}$ as the innermost and outermost cycles respectively.

The main result of this section can now be formally stated as follows.

Lemma 4.1. Let $G$ be a graph with $\operatorname{eg}(G)=g$, and $\operatorname{tw}(G) \geq 264 \cdot g \cdot \ell$. Then there exists a sequence of $\ell$-nested cycles in $G$.

\subsection{Reed's Result on Planar Punctured Graphs}

Let $\Sigma$ be a surface of Euler genus $g$. We denote by $\mathbf{b d}(\Sigma)$ the boundary of $\Sigma$, where each component of $\mathbf{b d}(\Sigma)$ is called a puncture. If $G$ is embedded in a surface $\Sigma$ with some punctures, we call such a surface a punctured surface. We now recall some definitions from $[21,25]$.

Let $\Sigma(a, b, c)$ denote the surface obtained from a 2-sphere by adding $a$ (anti)-handles, $b$ cross-caps, and $c$ punctures. Thus $\Sigma(0,0,1)$ is a closed disk, $\Sigma(0,0,2)$ is a cylinder, $\Sigma(1,0,0)$ is a torus, $\Sigma(0,1,0)$ is a projective plane, and $\Sigma(0,1,1)$ is a Möbius band. It is known that every connected surface is homeomorphic to $\Sigma(a, b, c)$ for some choice of $a, b, c$.

An $O$ - $\operatorname{arc}$ of $\Sigma$ is a simple (i.e. non-self-intersecting) closed noose of $\Sigma-\mathbf{b d}(\Sigma)$. An I-arc is a noose with both endpoints on $\mathbf{b d}(\Sigma)$. We also permit the degenerate case when the two endpoints coincide. An arc is proper if it intersects $G$ only at vertices. The length of a proper arc is the number of times it intersects $V(G)$.

We say that an $O$-arc $J$ surrounds a puncture $C$ if for some component $U$ of $\Sigma-J$ we have $C=U \cap \mathbf{b d}(\Sigma)$ (i.e., $U$ is $\Sigma(0,0, c)$ with $c \geq 2$ and $C$ is a boundary). We also say that an $O$-arc $J$ is contractible if there is a component $U$ of $\Sigma-J$ that is a closed disk bounded by $J$ (i.e., $U$ does not contain any other puncture, and $U$ is $\Sigma(0,0,1)$ ). Since we use this notation, our definition on representativity is the length of a shortest non-contractible noose in the surface where the punctures are filled with disks (i.e., this noose could go through a disk).

Now we recall the definition of a "schism" from [21, 25]. A lollipop consists of a proper $O$-arc $J$ surrounding some puncture $C$ and a proper $I$-arc from $J$ to $\operatorname{bd}(\Sigma)-C$ whose interior is contained in $\Sigma-b d(\Sigma)-J$. Note that an $I$-arc is allowed to have its endpoints on

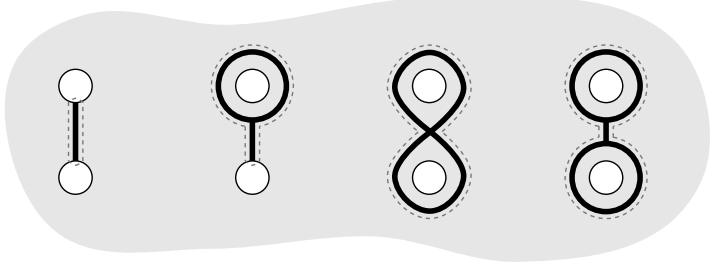

Figure 2: The four types of schisms: an $I$-arc, a lollipop, a butterfly, and a bicycle. The dashed lines indicate their length

an $O$-arc (and this also applies to the definitions below). A bicycle consists of two disjoint proper $O$-arcs $J$ and $J^{\prime}$ surrounding different punctures and a proper $I$-arc between them whose interior is contained in $\Sigma-\mathbf{b d}(\Sigma)-J-J^{\prime}$. A butterfly consists of two proper $O$-arcs, each surrounding a puncture, which meet at a single vertex. A schism is any of a lollipop, a bicycle, a butterfly, and a proper $I$-arc with its endpoints on different punctures. See Figure 2. The length of a schism is as described in Figure 2.

The following lemma is due to Reed [21], which builds on some results from [25].

Lemma 4.2 (Reed [21], Lemma 3). If $G$ is a graph embedded in a plane $\Sigma$ with at least 3 punctures, and $v$ is a vertex of $G$, then for any positive integer $\ell$, either

- $v$ is $\ell$-isolated,

- there is a proper simple arc $J$ with one endvertex $v$ and the other in $\mathbf{b d}(\Sigma)$ such that there are at most $\ell-1$ vertices on the interior of $\mathrm{J}$, or

- there is a proper $O$-arc $J_{1}$, and a proper I-arc $J_{2}$ with one endpoint $v$, the other on $J_{1}$, and no internal vertices on $J_{1}$ such that each component of $\Sigma-J_{1}$ contains at least one puncture and $\left|J_{1} \cap V(G)\right|+2\left|\left(J_{2}-J_{1}\right) \cap V(G)\right|<2 \ell$. (Note that we permit $J_{2}$ to be a single point in which case $J_{1}$ is simply an $\mathrm{O}$-arc through v.)

We also need the following result.

Lemma 4.3. Let $G$ be a graph embedded in a plane $\Sigma$ with $k$ punctures. If the treewidth of $G$ is at least $12 k \ell$, then there is an $\ell$-isolated vertex in $G$.

Proof. By Lemma 2.1 there is a $(t \times t)$ grid minor $W$, where $t=2 k \ell$. Since there are $k$ punctures, by the pigeonhole principle, there is a $(2 \ell \times 2 \ell)$ subgrid minor $W^{\prime}$ of $W$ that is contained in $\Sigma-\operatorname{bd}(\Sigma)$, and hence there is an $\ell$-isolated vertex in $W^{\prime}$.

\subsection{Extending to Surfaces}

We now extend Lemma 4.3 to graphs embedded in some punctured surface of large representativity. We first observe the following fact (see [20], Proposition 5.5.10).

FACT 4.4. If $G$ is embedded in a non-planar surface of representativity at least $4 l$, then there is a vertex that is $l$-isolated.

Note that this fact holds even if the underlying surface is nonorientable. We now show the following lemma. 
LEMMA 4.5. Suppose $G$ is embedded in a punctured non-planar surface $\Sigma$ of representativity at least $8 \ell$. Fix a puncture $C$. Let $U$ be the set of all vertices $v$ such that either

(i) there is a proper simple I-arc J with one endpoint $v$ and the other in $\operatorname{bd}(C)$ such that there are at most $\ell-1$ vertices on the interior of J, or

(ii) there is a proper $O$-arc $J_{1}$, and a proper I-arc $J_{2}$ with one endpoint $v$, the other on $J_{1}$, and no internal vertices on $J_{1}$ such that one component of $\Sigma-J_{1}$ is a punctured disk that contains the puncture $C$, and the other component contains $v$, and $\left|J_{1} \cap V(G)\right|+2\left|\left(J_{2}-J_{1}\right) \cap V(G)\right|<2 \ell$. Note that we permit $J_{2}$ to be a single point in which case $J_{1}$ is simply an $O$-arc through $v$.

Then the vertices in $U$ induce a planar graph, and there is no noncontractible noose that intersects only vertices in $U$.

Proof. We claim that there is no non-contractible noose $P$ that hits vertices only in $U$. Suppose that such a noose $P$ exists, and pick a shortest one. Note that the length of $P$ is at least $8 \ell$. We pick a pair of vertices $u, v$ of $P$ whose distance in $P$ is maximum, and thus must be at least $4 \ell$. As both $u$ and $v$ are in $U$, we have that both $u$ and $v$ satisfy one of the above conditions.

By combining two nooses as in (i) or (ii), it follows that either both $v$ and $u$ satisfy condition (ii) and the two nooses are disjoint, or there is an $I$-arc $P^{\prime}$ with endpoints $u$ and $v$ of length at most $4 \ell-1$ in the graph $G[U]$ (if the two nooses intersect). Note that in the second case every vertex in $P^{\prime}$ is in $U$, because it is obtained from either a proper $I$-arc or a proper $O$-arc from either (i) or (ii).

In the first case we now look at the noose $P$ again. It must intersect the $O$-arc $J_{1}^{\prime}$ that satisfies condition (ii) for either $u$ or $v$, at least twice. Thus we can obtain a noose $P^{\prime}$ which is part of $J_{1}^{\prime \prime}$ with endpoints $x, y$ such that $x, y$ are also in $P$, and moreover, there are at most $2 \ell-1$ internal vertices of $P^{\prime}$, all of them do not hit $P$.

Now by the 3-path condition (see Section 4.2 in [20]) $P \cup P^{\prime}$ contains a non-contractible noose which is shorter than that of $P$ in $G[U]$, a contradiction to our choice. Thus the vertices in $U$ induce a planar graph and there is no non-contractible noose that intersects only vertices in $U$.

Lemma 4.5 implies the following result. Due to lack of space, its proof appears in the full version of this paper.

Lemma 4.6. Let $G, C, U$ be as in Lemma 4.5 with $\ell$ replaced by $2 \ell$. Then there is a vertex $u \notin U$ that is adjacent to a vertex in $U$. Moreover, if the representativity of $G$ is at least $24 \ell$, then either there is a vertex $u$ that is $\ell$-isolated or there is a schism of order at most $12 \ell$.

\subsection{Main Proof}

We are now ready to show Lemma 4.1. Let us fix an integer $\ell$. Let us assume that $G$ is embedded in a surface $\Sigma$ (without punctures) of Euler genus $g$. We shall show that either there is an $\ell$-isolated vertex or $\operatorname{tw}(G) \leq 264 \cdot g \cdot \ell$.

To this end, we shall perform some cuts on $\Sigma$ that allow us to reduce the problem to several simpler subproblems. Note that we may divide $\Sigma$ into several components during this reduction. When this process is complete, each component is embedded in either a punctured sphere or a surface of representativity at least $24 \ell$. In this case either there is an $\ell$-isolated vertex or we can conclude that $\operatorname{tw}(G) \leq 264 \cdot g \cdot \ell$.

If a component $G_{i}$ of $G$ is embedded in a surface $\Sigma\left(a_{i}, b_{i}, c_{i}\right)$, we bound the quantity $2 a_{i}+b_{i}+c_{i}$, where $a_{i}$ is the number of handles, $b_{i}$ is the number of crosscaps, and $c_{i}$ is the number of punctures. Firstly, we note that $2 a+b+c \leq 2 g$ when we start with $G$ embedded in $\Sigma$ because $\Sigma(a, b, c)$ satisfies $c=0$ and $2 a+b \leq 2 g$, where $a, b, c$ for $G$ are defined in the same way as $a_{i}, b_{i}, c_{i}$ for $G_{i}$, respectively. Below we shall show that $\sum_{i} 2 a_{i}+b_{i}+c_{i} \leq 14 \mathrm{~g}$ whenever we reduce to several components $\left\{G_{i}\right\}_{i}$.

We shall perform the following algorithm.

Step 1. High representativity of an optimal embedding. If $G$ is embedded in a surface of Euler genus $g$ (without punctures) and the representativity of $G$ in $\Sigma$ is at least $24 \ell$, then there is an $\ell$-isolated vertex by Fact 4.4 , and hence we are done. Otherwise, the representativity of $G$ in $\Sigma$ is less than $24 \ell$, and we proceed to Step 2 (note that we consider Step 1 only once).

Step 2. Low representativity.

Suppose the representativity of the current graph $G$ in $\Sigma$ is

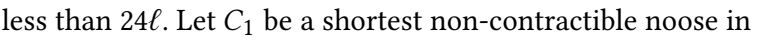
$G$. Assume first that $C_{1}$ is one-sided or surface-separating in $\Sigma$. We now cut the graph $G$ and the surface $\Sigma$ along the noose $C_{1}$ as described in [20], pp. 105-107. Note that each of these nooses is a boundary in the resulting graph which is embedded in a surface $\Sigma_{1}$ of Euler genus $g-1$ (if $C_{1}$ is one-sided) or embedded in two disjoint surfaces $\Sigma_{1}, \Sigma_{2}$, each of Euler genus less than $g$, whose sum of Euler genera is $g$ (if $C_{1}$ is surface-separating). In the resulting graph $G^{\prime}$, the cycle $C_{1}$ corresponds to a puncture $C_{1}^{\prime}$ (if $C_{1}$ is one-sided) or to two punctures $C_{1}^{\prime}$ and $C_{1}^{\prime \prime}$, respectively (if $C_{1}$ is surfaceseparating). Note also that we may obtain $G$ from $G^{\prime}$ by either identifying the opposite edges of $C_{1}^{\prime}$ (thus creating a crosscap) or identifying $C_{1}^{\prime}$ and $C_{1}^{\prime \prime}$.

Assume next that $C_{1}$ is surface-non-separating and twosided. Then the we cut along $C_{1}$, and we obtain two punctures, $C_{1}^{\prime}$ and $C_{1}^{\prime \prime}$, that correspond to the two sides of $C_{1}$.

For each component of $\Sigma-C_{1}$ that is still embedded in a nonsphere surface, we shall do the following: as long as the representativity of the current graph in the current punctured non-sphere surface $\Sigma(a, b, c)$ is less than $24 \ell$, we cut along a shortest noncontractible noose, as above. When the representativity of the current graph in a non-sphere $\Sigma(a, b, c)$ is at least $24 \ell$, we proceed to Step 3. For each component of $\Sigma-C_{1}$ that ends up with a punctured sphere, we stop.

Step 3. High representativity.

Suppose that the representativity of $G$ in $\Sigma$ is at least $24 \ell$. If there is a schism $W$ of order at most $12 \ell$, then we cut along this schism and reduce to several problems in a surface $\Sigma\left(a^{\prime}, b^{\prime}, c^{\prime}\right)$ simpler than $\Sigma(a, b, c)$, which means that either $a^{\prime}<a$, or $b<b^{\prime}$ and $a=a^{\prime}$, or $c^{\prime}<c, a^{\prime}=a$ and $b^{\prime}=b$. We then perform Step 2 for each component of $\Sigma-W$. If there is no schism of order at most $12 \ell$ in $G$, then we stop.

This completes the description of the algorithm. As mentioned in Step 1, if we end up with Step 1, we are done. Assume that from Step 3, some component ends up with a punctured surface which is 
not a sphere, and there is no short schism in it. Let $C$ be a puncture. By Lemma 4.6 either there is a vertex $u$ which is $\ell$-isolated, or there is a schism of order at most $12 \ell$. If the first happens, we are done. On the other hand the second does not suppose to happen.

Thus it remains to consider the case when each component comes from Step 2, and hence it is embedded in a punctured sphere. For this case, we need the following: Let $j_{1}$ and $j_{2}$ be the total number of times that we perform Step 2 and Step 3, respectively. Let $G_{1}, \ldots, G_{q}$ be the components obtained from this algorithm. We show the following:
(a) $j_{1} \leq 2 g-2$,
(b) $j_{2} \leq 2 j_{1}$,
(c) $q \leq j_{1}+j_{2}$, and
(d) $\sum_{i=1}^{q}\left(2 a_{i}+b_{i}+c_{i}\right) \leq 2 g+2 j_{1}+2 j_{2}$.

Let us show (a)-(d). For (a), since $\operatorname{eg}(G)=g$ the surface-separating noose case happens only $g-1$ times. Moreover, each non-contractible, non-surface-separating noose reduces the Euler genus by at least one. Thus there are at most $g-1$ times to find such a noose. So in total, $j_{1} \leq 2 g-2$.

Let us show (b). Since the schism we take in Step 3 is of length at most $12 \ell$ and representativity is at least $24 \ell$, every proper $O$-arc in this schism $W$ bounds a punctured disk that contains at least one puncture. Since $W$ can be thought of a closed noose of length at most $12 \ell$, by the definition of a schism, either (1) the interior of $W$ contains at least two punctures or (2) $W$ joins two different punctures by a proper $I$-arc or (3) the interior of $W$ contains at least one puncture and some other puncture is joined by a proper $I$-arc which is a part of $W$. Since the schism can be perturbed in a closed curve so that the resulting curve becomes a disk that bounds the punctures in the interior of $W$, it follows that $\Sigma-W$ consists of exactly two components such that one component is a punctured disk that contains at least two punctures, and hence the number of punctures (excluding the puncture obtained from $W$ ) in the other component decreases by at least two.

For the first component of the punctured disk, we stop. We apply this algorithm again, only to components that are not punctured disks. Note that the number of punctures in them decreases by at least one because we create only one new puncture that is obtained from $W$. So in order to bound $j_{2}$, it remains to show how many punctures in total we can obtain from Step 2, because in each time we apply Step 3, we reduce the number of punctures. As explained in Step 2, the noose in Step 2 creates at most two punctures. Thus, we obtain at most $2 j_{1}$ punctures from Step 2 , and hence $j_{2} \leq 2 j_{1}$.

For (c), since both the noose in Step 2 and the schism $W$ in Step 3 create at most two components (so we can increase the number of components by at most one), $q \leq j_{1}+j_{2}$.

Finally we show (d). We prove by induction on $j_{1}+j_{2}$. When $j_{1}+j_{2}=0$ we are done because $2 a+b+c \leq 2 g$ and there is only one component of $G$ (i.e., $G$ is embedded in a surface (without punctures) of $\operatorname{eg}(G)=g$ ). Assume $j_{1}+j_{2} \geq 1$. We must consider both Step 2 and Step 3.

Let us first look at Step 2. So assume that (d) holds for $j_{1}+\left(j_{2}-1\right)$. For simplicity, let $G$ be the component we consider now, and assume $\operatorname{eg}(G)=g$. As explained in Step 2 , if $C_{1}$ is one-sided, we reduce $b$ by one and we increase $c$ by one. If $C_{1}$ is surface-non-separating and two-sided in $\Sigma$, then we reduce $a$ by one and we increase $c$ by two.
In these two cases, we do not create any new component. Thus $q$ is the same, and hence $\sum_{i=1}^{q}\left(2 a_{i}+b_{i}+c_{i}\right) \leq 2 g+2\left(j_{1}-1\right)+2 j_{2}+2=$ $2 g+2 j_{1}+2 j_{2}$ by induction.

If $C_{1}$ is surface-separating in $\Sigma$, then we create a new component $G^{\prime}$ in $\Sigma-C_{1}$ and hence $q$ increases by one, but as explained in Step 2 , two components are embedded in two disjoint surfaces $\Sigma_{1}, \Sigma_{2}$, each of Euler genus less than $g$, whose sum of Euler genera is $g$. Moreover, in each component, we create only one new puncture that is obtained from $C_{1}$. Thus $\sum_{i=1}^{q+1}\left(2 a_{i}+b_{i}+c_{i}\right) \leq 2 g+2\left(j_{1}-\right.$ 1) $+2 j_{2}+2=2 g+2 j_{1}+2 j_{2}$.

Let us look at Step 3. So assume that $(\mathrm{d})$ holds for $\left(j_{1}-1\right)+j_{2}$. For notational simplicity, let $G$ be the component we consider at this step. As mentioned in the proof of (b), given a schism $W$ in Step 3, $\Sigma-W$ consists of exactly two components such that one component is a punctured disk that contains at least two punctures, and the number of punctures in the other component (excluding the puncture obtained from $W$ ) decreases by at least two. Moreover we create only two new punctures that are obtained from $W$. Thus $\sum_{i=1}^{q+1}\left(2 a_{i}+b_{i}+c_{i}\right) \leq 2 g+2 j_{1}+2\left(j_{2}-1\right)+2=2 g+2 j_{1}+2 j_{2}$. This proves (d).

We are now ready to complete the proof of Lemma 4.1. We only have to consider the case where each component ends up with a punctured sphere $\Sigma$. By (a)-(d), the number of punctures is at most $2 g+2 j_{1}+2 j_{2} \leq 2 g+6 j_{1} \leq 14 g-12$. In Step 2, we create at most two punctures by cutting along $C_{1}$ of at most $24 \ell$ vertices. In Step 3 , we create at most two punctures by cutting along $W$ of at most $12 \ell$ vertices. Thus there are at most $24 \ell \cdot j_{1}+12 \ell \cdot j_{2}<96 \mathrm{~g} \cdot \ell$ vertices $L$ of the original graph $G$ that are in some puncture. Moreover $G \backslash L$ can be embedded in a punctured sphere with at most $14 \mathrm{~g}$ punctures. By Lemma 4.3 either there is an $l$-isolated vertex or else $\operatorname{tw}(G \backslash L) \leq 168 g \cdot \ell$. In the second case, $\operatorname{tw}(G) \leq 264 g \cdot \ell$. This completes the proof of Lemma 4.1.

\section{ISOLATED VERTICES ARE IRRELEVANT}

In this section we prove that an $r$-isolated vertex, for sufficiently large $r$, is irrelevant. We begin with an auxiliary fact. Its proof appears in the full version of this paper.

Lemma 5.1. Let $G$ be a planar graph of maximum degree $\Delta$. Fix a planar drawing of $G$, and let $\mathcal{F}$ be a collection of faces of $G$. Then there exists some $\mathcal{F}^{\prime} \subseteq \mathcal{F}$, with $\left|\mathcal{F}^{\prime}\right| \geq|\mathcal{F}| /(20 \Delta)$, such that all the faces in $\mathcal{F}^{\prime}$ are pairwise vertex-disjoint.

We recall the following Lemma, which follows essentially from [9]. Here we use a slightly different terminology to match our exposition.

Lemma 5.2 (Juvan, MARInčEK AND Mohar [9]). Let $G$ be a 3connected planar graph and let $H$ be a r-isolated subgraph of $G$, for some $r \geq 2$. Then there exists a planar drawing $\phi$ of $G$ and $a$ sequence of pairwise vertex-disjoint cycles $C_{1}, \ldots, C_{r-1}$, satisfying the following conditions: (1) For every $i \in\{1, \ldots, r\}$, the disk bounded by $\phi\left(C_{i}\right)$ contains $\phi\left(C_{i-1}\right)$. (2) The disk bounded by $\phi\left(C_{2}\right)$ contains $\phi(H)$. (3) For any $i \in\{1, \ldots, r\}$, and for any component $J$ of $G \backslash\left(C_{1} \cup\right.$ $\left.\ldots \cup C_{r}\right)$, $J$ is not a $C_{i}$-bridge.

Let $G$ be a graph and let $H$ be a subgraph of $G$. We say that $H$ is irrelevant if $\operatorname{eg}(G \backslash H)=\operatorname{eg}(G)$. 
Lemma 5.3. Assume that $G$ is 2-connected, and let $H \subset G$ be connected and $80 \Delta \log n$-isolated. Then $H$ is irrelevant. Moreover, for any embedding of $\psi^{*}$ of $G$ into a surface of Euler genus $\operatorname{eg}(G)$, the innermost cycle bounding $H$ bounds a disk in $\psi^{*}$.

Proof. Let $g=\operatorname{eg}(G)$. Since $H$ is $r$-isolated, where $r=80 \Delta \log n$, it follows that there exist cycles $C_{1}, \ldots, C_{r}$ in $G$, such that $C_{r}$ is separating in $G$; moreover, if we let $C$ be the connected component of $G \backslash C_{r}$ containing $H$, and we set $\Gamma=G\left[C \cup C_{r}\right]$, then the graph $\Gamma$ is planar, and there exists some planar drawing $\phi$ of $\Gamma$ with outer face $C_{r}$, and such that if we let for each $i \in\{1, \ldots, r\}, \mathcal{D}_{i}$ be the disk bounded by $\phi\left(C_{i}\right)$, then $\phi(H) \subset \mathcal{D}_{1} \subset \ldots \subset \mathcal{D}_{r}$. Furthermore, by Lemma 5.2 we may assume that for any $i \in\{1, \ldots, r\}$, and for any component $J$ of $G \backslash\left(C_{1} \cup \ldots \cup C_{r}\right), J$ is not a $C_{i}$-bridge. Let $G^{\prime}$ be the subgraph of $G$ that is mapped into $\mathcal{D}_{r}$. If $G^{\prime}$ contains any 1-separator $x$, and $A$ is a connected component of $G \backslash x$ that does not contain $C_{r}$, then we delete $A$ from $G$; if $H \subseteq A$ then we set $H=x$. Similarly, if $G^{\prime}$ contains a 2-separator $\{x, y\}$ and $A$ is a component of $G \backslash\{x, y\}$, then we replace $A$ by the edge $\{x, y\}$; if $H \subseteq A$ then we set $H=x$. Repeating the above process, we arrive at a graph $G$ such that the corresponding graph $G^{\prime}$ is 3-connected, and $H$ remains $r$-isolated in $G$. Since we only delete planar pieces that are attached to the graph with 1-separators, and we replace by edges planar pieces that are attached via 2-separators, it is immediate that any embedding of the resulting graph into any surface can be extended to an embedding of the original graph into the same surface. Thus, $H$ is irrelevant in the new graph iff it is irrelevant in the original graph. Therefore, we may assume w.l.o.g. for the remainder of the proof that $G^{\prime}$ is 3-connected.

Let $\psi^{*}$ be an embedding of $G$ on a surface $\mathcal{S}$ of Euler genus eg $(G)$. Let $\psi$ be the induced embedding of $G^{\prime}$ on $\mathcal{S}$ obtained by restricting $\psi^{*}$ on $G^{\prime}$. For all $i \in\{1, \ldots, r\}$, since $C_{i}$ is separating in $G$, it follows that $\psi\left(C_{i}\right)$ is separating in $\mathcal{S}$. Therefore, if $\psi\left(C_{1}\right)$ bounds a disk in $\mathcal{S}$, it follows that $H$ is irrelevant, and there is nothing to show. It thus remains to consider the case where $\psi\left(C_{1}\right)$ does not bound a disk in $\mathcal{S}$. If there exists $i \in\{1, \ldots, r\}$ such that $\psi\left(C_{r}\right)$ bounds a disk, then we can obtain an embedding of $G$ into a surface of strictly smaller Euler genus by deleting the component of $\mathcal{S} \backslash \psi\left(C_{i}\right)$ that contains $\phi(H)$, and replacing it by a disk. Thus, we may also assume that for all $i \in\{1, \ldots, r\}, \psi\left(C_{i}\right)$ does not bound a disk. For each $i \in\{1, \ldots, r / 2\}$, let $\mathcal{N}_{i}$ be the set of $\phi$-facial cycles of $G^{\prime} \backslash H$ that are contained in $\mathcal{D}_{2 i} \backslash \mathcal{D}_{2 i-2}$, and are not $\psi$-facial, where we have used the notational convention $\mathcal{D}_{0}=\emptyset$. Since $r \geq 80 \Delta \log (n)$, and the total number of faces in the planar graph that can be embedded into the disk $\mathcal{D}_{r}$ is at most $2 n$ (by Euler's formula for planar graphs), it follows that there exists some $i^{*} \in$ $\{2, \ldots, r / 2\}$, such that $\left|\mathcal{N}_{i^{*}}\right|<\frac{1}{80 \Delta} \sum_{j=1}^{i^{*}-1}\left|\mathcal{N}_{j}\right|$. Let $\mathcal{N}=\bigcup_{i=1}^{i^{*}-1} \mathcal{N}_{i}$. By Lemma 5.1, there exists some $\mathcal{F}^{\prime} \subseteq \mathcal{N}$, such that all the faces in $\mathcal{F}^{\prime}$ are pairwise vertex-disjoint, with $\left|\mathcal{F}^{\prime}\right| \geq|\mathcal{N}| /(20 \Delta)$. Since $G^{\prime}$ is 3-connected, it follows that any $\phi$-facial cycle is non-separating in $G$. Therefore, for any $f \in \mathcal{F}^{\prime}$, the loop $\psi(f)$ is non-separating in $\mathcal{S}$. Furthermore, if there are $f_{1}, f_{2}, f_{3} \in \mathcal{F}^{\prime}$ such that $\psi\left(f_{1}\right), \psi\left(f_{2}\right)$, and $\psi\left(f_{3}\right)$ are pairwise homotopic in $\mathcal{S}$, then there must exist some cylinder $C \subset \mathcal{S}$, with boundary components $\psi\left(f_{1}\right)$ and $\psi\left(f_{3}\right)$ (say) and with $\psi\left(f_{2}\right)$ contained in the interior of $C$. However, this implies that $f_{1} \cup f_{3}$ separates $f_{2}$ form $C_{r}$ in $G$. Since $f_{1}, f_{2}$, and $f_{3}$ are $\phi$ facial, this can only happen if there exists some Jordan curve $\gamma$ in $\phi$ that separates $\phi\left(f_{2}\right)$ from $\phi\left(C_{r}\right)$, such that $\gamma$ is contained inside the disks bounded by $\phi\left(f_{1}\right)$ and $\phi\left(f_{3}\right)$. Thus, $\gamma$ intersects $f_{1} \cup f_{2}$ in a 2-separator, which contradicts the fact that $G^{\prime}$ is 3-connected. Therefore, we have established that at most two distinct faces in $\mathcal{F}^{\prime}$ are homotopic in $\mathcal{S}$. Therefore, there exists $\mathcal{F}^{\prime \prime} \subseteq \mathcal{F}^{\prime}$, with $\left|\mathcal{F}^{\prime \prime}\right| \geq\left|\mathcal{F}^{\prime}\right| / 2 \geq|\mathcal{N}| /(40 \Delta)$, such that for all distinct $f, f^{\prime} \in \mathcal{F}^{\prime \prime}$, we have that $\psi(f)$ and $\psi\left(f^{\prime}\right)$ are nonhomotopic in $\mathcal{S}$. Therefore, by cutting $\mathcal{S}$ along all the loops $\psi(f)$ for all $f \in \mathcal{F}^{\prime \prime}$, we obtain a surface $\mathcal{S}^{\prime}$ of genus at most $g-\left|\mathcal{F}^{\prime \prime}\right| \leq g-|\mathcal{N}| /(40 \Delta)$.

Let $Y=C_{2 i^{*}-1}$. Let $K \subseteq G^{\prime}$ be the graph mapped inside $\mathcal{D}_{2 i^{*}-1}$. Let $\mathcal{S}^{\prime \prime}$ be the surface obtained by cutting $\mathcal{S}$ along $\psi(K)$. Let $\mathcal{S}^{\prime \prime \prime}$ be the component of $\mathcal{S}^{\prime \prime}$ containing $\psi\left(G \backslash G^{\prime}\right)$. We remark that we delete $\psi(Y)$ from $\mathcal{S}^{\prime \prime \prime}$. Since $\mathcal{S}^{\prime \prime \prime} \subset \mathcal{S}^{\prime}$, it follows that $\mathcal{S}^{\prime \prime \prime}$ has genus at most $g-|\mathcal{N}| /(40 \Delta)$. Let $\mathcal{A}$ be the disk in the drawing $\phi$ with boundary $\phi(Y)$, containing $\phi(H)$. We shall connect the disk $\mathcal{A}$ to the surface $\mathcal{S}^{\prime \prime \prime}$. We consider two cases:

(1) $\psi(Y)$ is separating. We first observe that Lemma $5.2 \mathrm{im}-$ plies that $G \backslash Y$ consists of exactly two components such that one component $C$ is exactly drawn in the $\mathcal{A}$. Therefore $\psi(Y)$ separates $C$ from the rest of the graph. In this case cutting $\mathcal{S}$ along $\psi(Y)$ results in a puncture. We simply identify the boundary of $\mathcal{A}$ with this puncture.

(2) $\psi(Y)$ is non-separating and two-sided. Thus $\mathcal{S}^{\prime \prime \prime}$ has two punctures that correspond to the loop $\psi(Y)$. Let $Z_{1}$ and $Z_{2}$ be these two punctures. By the choice of the cycles $C_{1}, \ldots, C_{r}$, we have that no component of $G \backslash\left(C_{1} \cup \ldots \cup C_{r}\right)$ is a $Y$-bridge. This implies that in the drawing $\phi$, every face can intersect $Y$ in at most one (possibly empty) subpath. Therefore, the faces in $\phi$ that intersect $Y$ are ordered according to a traversal of $Y$. In the surface $\mathcal{S}^{\prime \prime \prime}$, some of these faces are incident to $Z_{1}$, and the rest are incident to $Z_{2}$. Traversing $Y$ in a clock-wise order, the number of times that the incident faces alternate between $Z_{1}$ and $Z_{2}$ is equal to the number of faces in $\phi$ that intersect $Y$ and are not facial in $\psi$. Thus, we can connect $\mathcal{S}$ to $\mathcal{S}^{\prime \prime \prime}$ by introducing at most one handle or anti-handle for each such alternation (see Figure 3).

(3) $\psi(Y)$ is non-separating and one-sided. The surface $\mathcal{S}^{\prime \prime \prime}$ has only one puncture corresponding to $\psi(Y)$, that contains the concatenation of two copies of $\psi(Y)$. In the surface $\mathcal{S}^{\prime \prime \prime}$, some of the faces in $\phi$ that intersect $Y$ are incident to the first copy, and the rest are incident to second copy. Traversing $Y$ in a clock-wise order, the number of times that the incident faces alternate between the two copies is equal to the number of faces in $\phi$ that intersect $Y$ and are not facial in $\psi$. Thus, similarly to case (2) above, we can connect $\mathcal{S}$ to $\mathcal{S}^{\prime \prime \prime}$ by introducing at most one handle or anti-handle for each such alternation.

Each handle or anti-handle added above increases the Euler genus of the resulting surface by at most 2 . We thus obtain an embedding of $G$ into a surface of Euler genus at most $g-|\mathcal{N}| /(40 \Delta)+2\left|\mathcal{N}_{i^{*}}\right|<$ $g$, which contradicts the fact that $\operatorname{eg}(G)=g$, and concludes the proof. 

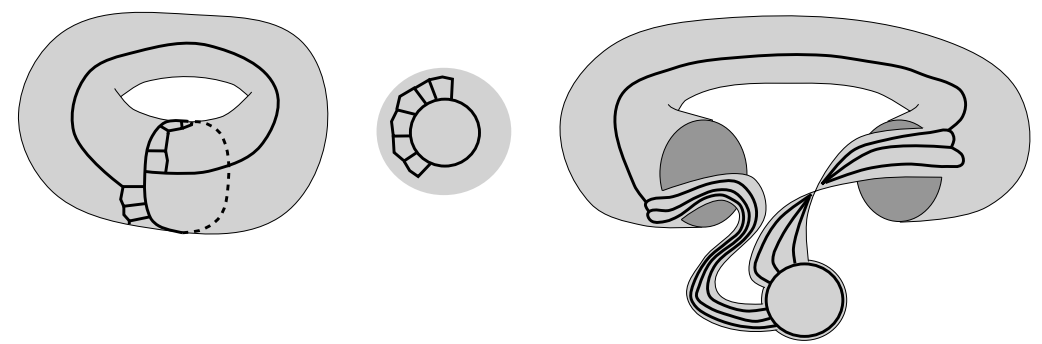

Figure 3: Example of the construction in the proof of Lemma 5.3: An optimal embedding of $G$ into a surface $\mathcal{S}$ (left), the planar embedding $\phi$ on $Y$ inside a disk $\mathcal{A}$ and its incident faces (middle), and the result of attaching $\mathcal{A}$ to $\mathcal{S}^{\prime \prime \prime}$ (right). The loop $\psi(Y)$ is two-sided, and thus cutting along $\psi(Y)$ we obtain two punctures. The figure depicts the attachment obtained by adding one handle and one anti-handle between $\mathcal{A}$ and $\mathcal{S}^{\prime \prime \prime}$.

\section{COMPUTING AN ISOLATED VERTEX}

In this section we show how to efficiently compute an $\ell$-isolated vertex, if it exists.

Lemma 6.1. Let $G$ be a 3-connected graph. Suppose that there is a sequence of $\ell$ nested cycles in $G$, for some $\ell>2 \log n$. Then, in polynomial time, we can compute a vertex $v$ that is $\ell / 2$-isolated, and its $\ell / 2$ nested cycles.

Remark: Let us observe that in our whole algorithm we may assume that $G$ is 3-connected. To see this, as in the proof of Lemma 5.3, if $G$ contains any 1-separator $x$, and $A$ is a connected component of $G \backslash x$ that induces a planar graph, then we delete $A$ from $G$. Similarly, if $G$ contains a 2-separator $\{x, y\}$ and $A$ is a component of $G \backslash\{x, y\}$, then we replace $A$ by the edge $\{x, y\}$. Repeating the above process, we arrive at a graph $G$ such that the corresponding graph $G^{\prime}$ is 3-connected. Since we only delete planar pieces that are attached to the graph with 1-separators, and we replace by edges planar pieces that are attached via 2-separators, it is immediate that any embedding of the resulting graph into any surface can be extended to an embedding of the original graph into the same surface. So we may assume that $G$ is 3-connected.

We also remark that the condition " $\ell>2 \log n$ " is critical as we will see in the proof below. Roughly speaking, whenever we can find a cycle within distance $2 \ell$, we can find a partial embedding, as mentioned in (2) below. It turns out that the worst case is a "binary tree" of depth $\log n$ (i.e., no cycle in the end), which implies that this tree has at least $n$ vertices. See at the end of the proof.

Proof. Suppose that there exist $\ell$ nested cycles $K_{1}, \ldots, K_{\ell}$. For each $i \in\{2, \ldots, \ell-1\}$, we have that $K_{i}$ separates $K_{1} \cup \ldots \cup K_{i-1}$ from $K_{i+1} \cup \ldots \cup K_{\ell}$ in $G$. For each $i \in\{1, \ldots, \ell-1\}$ let $U_{i}=\{u \in$ $V(G): K_{i}$ separates $u$ and $V\left(K_{\ell}\right)$ in $\left.G\right\}$, and $\Gamma_{i}=G\left[U_{i}\right]$. We further assume that $\Gamma_{1}$ contains a vertex $v$. Below, we assume that we find the vertex $v$ by brute-forcing.

We first modify the nested cycles $K_{1}, \ldots, K_{\ell}$ as follows. In $H=$ $\Gamma_{\ell} \cup K_{\ell}$ which is embedded in a plane with the outer face boundary $K_{\ell}$, let us construct the radial graph $H^{*}$, which is the bipartite graph with sides $V(H)$ and $F(H)$, where $F(H)$ is a set of faces of the embedded graph $H$, such that $v \in V(H)$ is adjacent to $f \in F(H)$ iff $v$ is contained in $f$. Note that $H^{*}$ is a planar graph. We first establish the following:
(1) We can modify the nested cycles $K_{1}, \ldots, K_{\ell}$ so that every vertex in $K_{i}$ is co-facial with a face whose corresponding vertex in $H^{*}$ is of distance exactly $2 i-1$ from $v$ in $H^{*}$.

This can be shown by induction on $i$. For $i=1, K_{1}$ is obtained from the resulting face of deleting $v$. Note that since $G$ is 3-connected, such a resulting face exists. Thus every vertex in $K_{1}$ is co-facial with $v$ and hence the result holds.

For the induction step, suppose that (1) holds for $i-1$, for some $i \geq 2$. Let us consider the cylinder $C$ bounded by $K_{i-1}$ and $K_{i}$. Let $S=K_{i-1} \cup K_{i}$. We consider a nontrivial $S$-bridge $B$ that contains only vertices in the cylinder $C$. If every such a bridge $B$ has either at most one attachment in $K_{i}$ or exactly two attachments that are adjacent in $K_{i}$, then every vertex in $K_{i}$ is co-facial with a vertex in $K_{i-1}$, and hence by induction, every vertex in $K_{i}$ is co-facial with a face whose corresponding vertex in $H^{*}$ is of distance exactly $2 i-1$ from $v$ in $H^{*}$.

So suppose that there is a $S$-bridge $B$ that has either at least three attachments in $K_{i}$ or exactly two attachments that are not adjacent in $K_{i}$. We call such a bridge $b a d$. Let us take $K_{i}$ such that the number of vertices in bad bridges is as small as possible. If there is a bad bridge $B$, we can reroute $K_{i}$ so that it contains at least one vertex in $B$ (and contains no vertex of $K_{i-1}$ ). Let $K_{i_{1}}$ be the resulting cycle of $K_{i}$. By the planarity of the cylinder $C$, no vertex in $V\left(K_{i}\right) \backslash V\left(K_{i_{1}}\right)$ is an attachment of any such an $S$-bridge. But this contradicts our choice of $K_{i}$, and hence there is no such a bad bridge. Therefore (1) holds. Note that during the process, we only make the graph $\Gamma_{i}$ smaller.

Remark: The proof of (1) is constructive in the sense that it can be converted to a polynomial time algorithm to construct $\ell$ nested cycles so that every vertex in $K_{i}$ is co-facial with a face whose corresponding vertex in $H^{*}$ is of distance exactly $2 i-1$ from $v$ in $H^{*}$.

Thus (1) implies that in order to detect $\ell$ nested cycles for $v$, it suffices to find all the vertices whose distance in $H^{*}$ is at most $2 \ell$. Next, we establish another useful property:

(2) Assume that we detect a cycle $C$ in $\Gamma_{\ell}$. Then in polynomial time either we can decide that $C$ is a face in the (unknown) optimal embedding of $\Gamma_{\ell} \cup K_{\ell}$ or we can obtain a planar embedding of a subgraph that contains $C$, such that every $C$-bridge that does not contain a vertex in $G-\Gamma_{\ell}$ is embedded. Moreover, this embedding 
is consistent with the (unknown) optimal embedding of $\Gamma_{\ell} \cup K_{\ell}$, up to Whitney flips.

If there is a $C$-bridge $B$ that contains a vertex in $K_{\ell}$, then it contains all the vertices of $K_{\ell}$, and hence it contains a vertex in $G-\Gamma_{\ell}$. Moreover, there is no other $C$-bridge $B^{\prime}$ that contains a vertex in $G-\Gamma_{\ell}$, for otherwise, $B^{\prime}$ must contain a vertex in $K_{\ell}$. Let us call the unique bridge $B$ special. Let $\mathcal{B}$ be the set of all $C$-bridges that are not special. Every vertex contained in a bridge in $\mathcal{B}$ is in $\Gamma_{\ell}$, and thus all the bridges in $\mathcal{B}$, together with $C$, can be embedded in the plane. Moreover, we can find such an embedding by a planarity testing algorithm in linear time. Clearly this embedding is consistent with the (unknown) optimal embedding of $\Gamma_{\ell} \cup K_{\ell}$, up to Whitney flips, because $G$ is 3-connected. If $\mathcal{B}=\emptyset$, then we conclude that $C$ is a face in $\Gamma_{\ell}$. This proves (2).

Remark: The proof of (2) implies that we can embed all the non-special $C$-bridges in a plane. In this case $C$ may not be the outer face boundary, because we may perform Whitney flips.

Property (2) implies that whenever we find a cycle in $\Gamma_{\ell}$, we can make progress toward finding the optimal (but unknown) planar embedding of $\Gamma_{\ell} \cup K_{\ell}$.

At the high level, our algorithm proceeds as follows. Let $H^{*}$ be as in (1). We want to compute all the vertices of distance at most $\ell$ from $v$ in $H^{*}$. If we can do that, then by (1) (and the argument used in its proof), we can construct, in polynomial time, $\ell / 2$ nested cycles so that every vertex in $K_{i}$ is co-facial with a face whose corresponding vertex in $H^{*}$ is of distance exactly $2 i-1$ from $v$. Therefore, it remains to detect the graph $H^{*}$.

To this end, we first apply BFS to find all the vertices of distance (in $G$ ) $\ell$ from $v$. Note that at the beginning, we cannot define the radial graph because we do not have any embedding, even any partial embedding. Then we apply (2) whenever we obtain a cycle in $\Gamma_{\ell}$. Since by (2) the embedding we obtain here is consistent with the (unknown) optimal embedding of $\Gamma_{\ell} \cup K_{\ell}$, up to Whitney flips, this allows us to define the "radial graph" to the embedded subgraph. Therefore we can update the distance of some vertices of $G$ via the radial graph.

To summarize, at the beginning, the distance is measured in the original graph. In the end we want to measure the distance in terms of the radial graph. So the partial embedding of some subgraph allows us to update the distance in the constructed radial subgraph. This is the intermediate step toward figuring out the distance in the radial graph of the (unknown) optimal embedding of $\Gamma_{\ell} \cup K_{\ell}$.

Note that every vertex in the radial graph is at even distance from $v$, and half the distance in the radial graph may be smaller than that in the original graph (but not larger). Since, after the update of distance, we may obtain smaller distance for some vertices in the radial graph we have constructed so far (than that in the previous iteration), we can apply BFS again to explore more vertices. It turns out that this process does not stop until we detect all the vertices at distance $\ell$ from $v$ in $H^{*}$ (and hence we are done if we replace $\ell$ in (1) by $\ell / 2)$.

Let us now describe the algorithm in more detail. Again, we have already fixed the vertex $v$.

Step 1. Exploring vertices. Given a graph $A$ that is contained in the (unknown) graph $\Gamma_{\ell}$, we do the following. Each vertex $u$ in $A$ has label $L_{u}$, and we take all the vertices $v^{\prime}$ that is of distance at most $\ell-L_{u} / 2-1$ in $G \backslash A$. We do this process for all the vertices in $A$, and we let $W$ be the graph induced by $A$ together with all the vertices in $V(G) \backslash V(A)$ we obtain from this process (for all the vertices in $A$ ).

Initially $A=\{v\}, L_{v}=0$.

If $W$ contains all the vertices in $\Gamma_{\ell / 2} \cup K_{\ell / 2}$, where the embedding of $\Gamma_{\ell / 2} \cup K_{\ell / 2}$ is constructed in $W$ then we stop. Then apply the algorithm of (1) to detect the nested cycles $K_{1}, \ldots, K_{\ell / 2}$.

Otherwise we proceed to Step 2.

Step 2. Finding a cycle. We find a cycle $C$ in $W$ that has not been detected so far. We apply (2) to the cycle $C$, and we obtain a planar embedding of some induced subgraph $Q$ that contains $V(C)$. When applying (2), we have only one special $C$-bridge in $G$ as in the proof of (2), so we just need to embed all the $C$-bridges, except for one which can be determined as the "special" bridge.

Let $C^{\prime}$ be the outer face boundary of $Q$ (as remarked after the proof of (2), $C^{\prime}$ could be different from $C$ ). Delete the vertices inside the disk $D^{\prime}$ bounded by $C^{\prime}$, and let $W$ be the resulting graph. We repeat Step 2 until no new cycle is found.

By (2), any new cycle we detect here yields either a face or a planar embedding of some induced subgraph that is consistent with the (unknown) optimal embedding of $\Gamma_{\ell} \cup K_{\ell}$ (up to Whitney flips).

Note that Step 2 is the only step to construct an embedding, and we ensure that the embedding constructed here is always consistent with the (unknown) optimal embedding of $\Gamma_{\ell} \cup K_{\ell}$, up to Whitney flips. We next proceed to Step 3.

Step 3. Constructing the radial graph. Let $A^{\prime}$ be the graph embedded in a plane which is obtained from $W$ in Step 2, together with the embedded graph inside the disk $D^{\prime}$ for each $D^{\prime}$ (note that we delete it in Step 2, and here we put it back to the embedding). Now $A^{\prime}$ induces a tree-decomposition $(T, R)$ such that each $R_{t}$ either is a singleton or consists of a 2-connected plane induced subgraph $\left(R_{t}, C^{\prime \prime}\right)$ with the outer face boundary $C^{\prime \prime}$. Moreover, for any edge $r r^{\prime} \in T, R_{r} \cap R_{r^{\prime}}$ is a singleton (i.e., the tree-decomposition is constructed by just detecting cut vertices of $A^{\prime}$ ).

For each 2-connected plane induced subgraph $\left(R_{t}, C^{\prime \prime}\right)$, we construct the radial graph $R_{t}^{*}$, omitting the vertex that corresponds to the outer face boundary $C^{\prime \prime}$. We next proceed to Step 4.

Step 4. Modifying the radial graph by "half" faces. Let us again look at the plane induced subgraph $\left(R_{t}, C^{\prime \prime}\right)$. For each segment $P$ of $C^{\prime \prime}$ with two endvertices $u_{1}, u_{2}$ and $|P| \geq 3$ such that all the internal vertices in $P$ have no neighbor outside $R_{t}$ in $G$, we add a dummy vertex $p$ to $R_{t}^{*}$ such that $p$ is adjacent to all the vertices of the radial graph $R_{t}^{*}$ whose corresponding vertices are in $P$.

We do this for each node $t \in T$ (and $R_{t}$ ). Let $A^{*}$ be the resulting graph obtained from constructing radial graphs $R_{t}^{*}$ and adding the dummy vertices, and let $Y$ be all the dummy vertices we added here. Note that in the previous step, we might have already added some dummy vertices, but some dummy vertex $v^{\prime \prime}$ may be gone at this step, if we detect 
the face that contains $v^{\prime \prime}$ in Step 2. Note also that if $R_{t}$ is a singleton, it is still a singleton in $A^{*}$. Then we proceed to Step 5.

Step 5. Updating the labeling. We update a label to each vertex of $A^{\prime}$ as follows: each vertex $u$ in $A^{*}$ has label $L_{u}$ which is exactly the distance between $v$ and $u$ in $A^{*}$. The label of vertices of $A^{*}$ yields the label of the corresponding vertices of $A^{\prime}$. Note that if $u$ had some label $L_{u}^{\prime}$ previously, then $L_{u} \leq L_{u}^{\prime}$ because we only add some vertices (including dummy vertices) from the previous graph, which only results in shortening distance in $A^{*}$. We set $A=A^{\prime}$ with each vertex $u$ having label $L_{u}$, and we go to Step 1 .

This algorithm stops at Step 1 when the embedding of $\Gamma_{\ell / 2} \cup K_{\ell / 2}$ is constructed.

Let us show that each step runs in polynomial time. Step 1 clearly runs in polynomial time, because we only have to perform BFS from each vertex $u$. Note that the embedding of $\Gamma_{\ell / 2} \cup K_{\ell / 2}$ is already constructed, so we just apply the algorithm of (1) to detect the nested cycles $K_{1}, \ldots, K_{\ell / 2}$.

For Step 2, finding a cycle can be clearly done in polynomial time. (2) runs in linear time. Once we find one cycle $C$, the number of the vertices in the rest of the graph $\Gamma_{\ell}$ (i.e., vertices that are not yet embedded) decreases by at least one. Moreover, for any $C$-bridge $B$ that is not special (remind the definition of "special" in the proof of (2)), in the (unknown) optimal embedding of $\Gamma_{\ell} \cup K_{\ell}, B$ has to be embedded in the plane. Again, finding such an embedding of $B$ can be done in linear time by some planarity testing. So Step 2 runs in polynomial time.

Step 3 clearly runs in polynomial time because it only constructs the radial graph from an embedded graph. Note that $A^{\prime}$ is a treedecomposition such that for any edge $r r^{\prime} \in T, R_{r} \cap R_{r^{\prime}}$ is a singleton, i.e., the tree-decomposition is constructed by just detecting cut vertices of $A^{\prime}$.

In Step 4, if we have an embedding of a 2-connected plane graph $R_{t}$ with the outer face boundary $C^{\prime \prime}$, then we can easily find such a path $P$. Note that such a path $P$ implies that it must be contained in a face in the (unknown) optimal embedding of $\Gamma_{\ell} \cup K_{\ell}$. Thus adding the vertices $Y$ to the radial graph $A^{*}$ does not destroy the planarity of $A^{*}$. Moreover, no two vertices in $Y$ are adjacent. So clearly Step 4 runs in polynomial time.

In Step 5, again, we only have to perform BFS from $v$ in $A^{*}$ so it runs in polynomial time (note that $A^{*}$ has at most $|V(G)|+|F(G)|$ vertices and $|F(G)| \leq 2|V(G)|)$.

So our algorithm runs in polynomial time. In order to complete our proof of Lemma 6.1 it remains to show the following:

(3) Every vertex at distance at most $\ell$ from $v$ in $H^{*}$ is visited by the algorithm.

From (3) and (1), it immediately follows that we can find $\ell / 2$ nested cycles in polynomial time.

Let us now proceed the proof of (3). Assume, for the sake of contradiction, that there is a vertex $v^{\prime}$ in $G$ of distance $q<\ell$ from $v$ in $H^{*}$, that is not visited in our algorithm. Let $A^{\prime}$ be the resulting (partially embedded) graph obtained from this algorithm.

We have that there is an even number $i^{*}$ with $0 \leq i^{*} \leq \ell-1$ such that all the vertices of distance at most $i^{*}$ from $v$ in $H^{*}$ are detected in this algorithm, but this is not the case for $i^{*}+2$. Then the graph $\Gamma_{i^{*} / 2} \cup K_{i^{*} / 2}$ is contained in a 2-connected plane induced subgraph $\left(R_{t}, C^{\prime \prime}\right)$ in the tree-decomposition of $A^{\prime}$, and there is a vertex $u$ in $R_{t}$ and there is also a neighbor $u^{\prime}$ of $u$ in $V\left(A^{\prime}\right) \backslash V\left(R_{t}\right)$.

In Step 1, we have already taken all the vertices $Z$ (with $Z \cap$ $V\left(R_{t}\right)=\emptyset$ ) of distance at most $\ell-L_{u} / 2-1$ from $u$. Note that $L_{u}<\ell$. We claim that after contracting some $R_{t^{\prime}}$ for some $t^{\prime}$, into a single point, $Z$ induces a tree of depth more than $\ell / 2$ and of degree at least 3. To see this, firstly, if $R_{t^{\prime}}$ is not a singleton for some $t^{\prime}$, we can reach the vertex $R_{t_{1}} \cap R_{t^{\prime}}$ from the vertex $R_{t_{2}} \cap R_{t^{\prime}}$ by distance at most two in $A^{\prime}$, where $t_{1} t^{\prime}, t_{2} t^{\prime} \in T$ and $t_{2}$ is the parent of $t^{\prime}$ (i.e., we can find such a node $t_{1} \in T$. Note also that the parent $t_{2}$ is closer to $t$ in $T$ than $t^{\prime}$ ), because either a dummy vertex in the boundary of $R_{t^{\prime}}$ exists (which allows us to reach the vertex $R_{t_{1}} \cap R_{t^{\prime}}$ from the vertex $R_{t_{2}} \cap R_{t^{\prime}}$ by distance exactly two in $A^{\prime}$ ) or the vertex $R_{t_{1}} \cap R_{t^{\prime}}$ is of distance at most two from the vertex $R_{t_{2}} \cap R_{t^{\prime}}$ in $C^{\prime \prime}$. Indeed, if $t^{\prime}$ has at least two children, we can apply the above argument to find two such nodes $t_{1}$ by following one and the other direction of $C^{\prime \prime}$ from $R_{t_{2}} \cap R_{t^{\prime}}$. Since $G$ is 3-connected, it follows that each $R_{t}$ has at least two children. Thus $Z$ is a tree such that each vertex is of degree at least 3 , and depth is at least $\ell / 2$. Note that $Z$ does not have a vertex that has a neighbor in $R_{t}$ (otherwise we would have already detected this cycle in Step 2, which would imply that this cycle would be contained in $R_{t}$ ). Then $Z$ has at least $2^{\ell / 2}>n$ vertices, a contradiction. This concludes the proof.

Remark: From the proof of Lemma 6.1 it follows that if there is no sequence of $\ell$ nested cycles in $G$, for some $\ell>2 \log n$, then the algorithm in Lemma 6.1 simply tells us that "there is no vertex that is a $\ell / 2$-isolated".

\section{REFERENCES}

[1] Dan Archdeacon. 1981. A Kuratowski theorem for the projective plane. Fournal of Graph Theory 5, 3 (1981), 243-246. https://doi.org/10.1002/jgt.3190050305

[2] Chandra Chekuri and Anastasios Sidiropoulos. 2013. Approximation Algorithms for Euler Genus and Related Problems. In 54th Annual IEEE Symposium on Foundations of Computer Science, FOCS 2013, 26-29 October, 2013, Berkeley, CA, USA. 167-176. https://doi.org/10.1109/FOCS.2013.26

[3] Chandra Chekuri and Anastasios Sidiropoulos. 2013. Approximation algorithms for Euler genus and related problems. In Foundations of Computer Science (FOCS), 2013 IEEE 54th Annual Symposium on. IEEE, 167-176.

[4] Markus Chimani and Petr Hlinený. 2017. A tighter insertion-based approximation of the crossing number. F. Comb. Optim. 33, 4 (2017), 1183-1225. https://doi.org/ 10.1007/s10878-016-0030-z

[5] M. R. Garey and David S. Johnson. 1979. Computers and Intractability: A Guide to the Theory of NP-Completeness. W. H. Freeman.

[6] Henry H. Glover, John Philip Huneke, and Chin San Wang. 1979. 103 Graphs that are irreducible for the projective plane. F. Comb. Theory, Ser. B 27, 3 (1979), 332-370. https://doi.org/10.1016/0095-8956(79)90022-4

[7] John E. Hopcroft and Robert Endre Tarjan. 1974. Efficient Planarity Testing. F. ACM 21, 4 (1974), 549-568. https://doi.org/10.1145/321850.321852

[8] Martin Juvan, Joze Marincek, and Bojan Mohar. 1995. Embedding Graphs in the Torus in Linear Time. In Integer Programming and Combinatorial Optimization, 4th International IPCO Conference, Copenhagen, Denmark, May 29-31, 1995, Proceedings. 360-363. https://doi.org/10.1007/3-540-59408-6_64

[9] Martin Juvan, Jože Marinček, and Bojan Mohar. 1997. Elimination of local bridges. Mathematica Slovaca 47, 1 (1997), 85-92.

[10] Ken-ichi Kawarabayashi, Bojan Mohar, and Bruce A. Reed. 2008. A Simpler Linear Time Algorithm for Embedding Graphs into an Arbitrary Surface and the Genus of Graphs of Bounded Tree-Width. In 49th Annual IEEE Symposium on Foundations of Computer Science, FOCS 2008, October 25-28, 2008, Philadelphia, PA, USA. 771-780. https://doi.org/10.1109/FOCS.2008.53

[11] Ken-ichi Kawarabayashi and Anastasios Sidiropoulos. 2015. Beyond the Euler Characteristic: Approximating the Genus of General Graphs. In Proceedings of the Forty-Seventh Annual ACM on Symposium on Theory of Computing, STOC 2015, Portland, OR, USA, June 14-17, 2015. 675-682. https://doi.org/10.1145/2746539. 2746583 
[12] Ken-ichi Kawarabayashi and Anastasios Sidiropoulos. 2017. Polylogarithmic approximation for minimum planarization (almost). In Proceedings of the IEEE Symposium on Foundations of Computer Science, FOCS 2017.

[13] Tomasz Kociumaka and Marcin Pilipczuk. 2017. Deleting vertices to graphs of bounded genus. CoRR abs/1706.04065 (2017). arXiv:1706.04065 http://arxiv.org/ abs/1706.04065

[14] Casimir Kuratowski. 1930. Sur le probleme des courbes gauches en topologie. Fundamenta mathematicae 15, 1 (1930), 271-283.

[15] Yury Makarychev, Amir Nayyeri, and Anastasios Sidiropoulos. 2013. A Pseudoapproximation for the Genus of Hamiltonian Graphs. In Approximation, Randomization, and Combinatorial Optimization. Algorithms and Techniques - 16th International Workshop, APPROX 2013, and 17th International Workshop, RANDOM 2013, Berkeley, CA, USA, August 21-23, 2013. Proceedings. 244-259. https: //doi.org/10.1007/978-3-642-40328-6_18

[16] Bojan Mohar. 1993. Projective Planarity in Linear Time. f. Algorithms 15, 3 (1993), 482-502. https://doi.org/10.1006/jagm.1993.1050

[17] Bojan Mohar. 1996. Embedding Graphs in an Arbitrary Surface in Linear Time. In Proceedings of the Twenty-Eighth Annual ACM Symposium on the Theory of Computing, Philadelphia, Pennsylvania, USA, May 22-24, 1996. 392-397. https: //doi.org/10.1145/237814.237986

[18] Bojan Mohar. 1999. A Linear Time Algorithm for Embedding Graphs in an Arbitrary Surface. SIAM 7. Discrete Math. 12, 1 (1999), 6-26. https://doi.org/10 1137/S089548019529248X

[19] Bojan Mohar. 2001. Face Covers and the Genus Problem for Apex Graphs. F. Comb. Theory, Ser. B 82, 1 (2001), 102-117. https://doi.org/10.1006/jctb.2000.2026

[20] Bojan Mohar and Carsten Thomassen. 2001. Graphs on Surfaces. Johns Hopkins University Press. http://jhupbooks.press.jhu.edu/ecom/MasterServlet/
GetItemDetailsHandler?iN=9780801866890\&qty $=1 \&$ source $=2 \&$ viewMode $=3 \&$ loggedIN=false $\&$ JavaScript=y

[21] Bruce A. Reed. 1995. Rooted Routing in the Plane. Discrete Applied Mathematics 57, 2-3 (1995), 213-227. https://doi.org/10.1016/0166-218X(94)00104-L

[22] Gerhard Ringel. 1974. Map color theorem. Springer.

[23] Neil Robertson, Paul Seymour, and Robin Thomas. 1994. Quickly excluding a planar graph. Journal of Combinatorial Theory, Series B 62, 2 (1994), 323-348.

[24] Neil Robertson and Paul D. Seymour. 1986. Graph minors. V. Excluding a planar graph. F. Comb. Theory, Ser. B 41, 1 (1986), 92-114. https://doi.org/10.1016/ 0095-8956(86)90030-4

[25] Neil Robertson and Paul D. Seymour. 1988. Graph minors. VII. Disjoint paths on a surface. F. Comb. Theory, Ser. B 45, 2 (1988), 212-254. https://doi.org/10.1016/ 0095-8956(88)90070-6

[26] Neil Robertson and Paul D. Seymour. 1990. Graph minors. VIII. A Kuratowski theorem for general surfaces. 7. Comb. Theory, Ser. B 48, 2 (1990), 255-288.

[27] Neil Robertson and Paul D. Seymour. 1994. Graph Minors. XI. Circuits on a Surface. f. Comb. Theory, Ser. B 60, 1 (1994), 72-106. https://doi.org/10.1006/jctb.1994.1007

[28] Neil Robertson, Paul D. Seymour, and Robin Thomas. 1994. Quickly Excluding a Planar Graph. 7. Comb. Theory, Ser. B 62, 2 (1994), 323-348.

[29] Carsten Thomassen. 1989. The Graph Genus Problem is NP-Complete. F. Algorithms 10, 4 (1989), 568-576. https://doi.org/10.1016/0196-6774(89)90006-0

[30] Carsten Thomassen. 1997. The Genus Problem for Cubic Graphs. F. Comb. Theory, Ser. B 69, 1 (1997), 52-58. https://doi.org/10.1006/jctb.1996.1721

[31] Klaus Wagner. 1937. Uber eine Eigenschaft der ebenen Komplexe. Math. Ann. 114, 1 (1937), 570-590. 